\title{
Generation of Nano-Catalyst Particles by Spinodal Nano-Decomposition in Perovskite
}

\author{
Hidetoshi Kizaki*, Koichi Kusakabe, Soichiro Nogami, and Hiroshi Katayama-Yoshida \\ Graduate School of Engineering Science, Osaka University, Toyonaka, Osaka 567-8531, Japan
}

\begin{abstract}
A new mechanism of nano-catalyst generation based on the spinodal nano-decomposition in self-regenerating perovskite catalysts for automotive-emissions control is proposed. To demonstrate existence of the spinodal nano-decomposition in real perovskite catalysts, we performed first-principles calculations to evaluate the free energy of $\mathrm{La}\left(\mathrm{Fe}_{1-x} \mathrm{Pd}_{x}\right) \mathrm{O}_{3}$ and $\mathrm{La}\left(\mathrm{Fe}_{1-x} \mathrm{Rh}_{x}\right) \mathrm{O}_{3}$. The result indicates appearance of a spinodal region in the phase diagram of each material. Formation of nano-catalyst particles in the perovskite host matrix is crucial for the self-regeneration of perovskite catalyst. Based on the spinodal nano-decomposition model, possible materials are designed for new three-way catalyst with no contents of precious metal.
\end{abstract}

Self-regenerating Pd-, Rh-, and Pt-doped perovskite catalysts for automotive-emissions control are attracting much interest due to their unique functionalities. ${ }^{1-11)}$ In a conventional catalyst made of fine precious-metal particles supported on a solid like an almina, agglomeration of the metal particles is inevitable because of high temperature conditions in the redox environment. This is the very reason for deterioration of automotive three-way catalysts. In the self-regenerating perovskite catalysts, interestingly, the deterioration is strongly suppressed. Therefore, consumption of the precious metal is greatly reduced, providing a highly efficient solution for supply problems. The reason for non-deterioration is thought to be reformation of a precious-metal doped perovskite lattice in the $\mathrm{NO}_{x}$-reduction environment from segregated nano-particles of precious-metal created in the $\mathrm{CO}$ - and $\mathrm{C}_{x} \mathrm{H}$-oxidation environment. In this regeneration model, precious-metal atoms are assumed to move into and out of the perovskite host matrixes. Growth of precious-metal grains is suppressed due to this repeated motion of precious metal atoms between a solid solution and metallic nano-particles during three-way catalytic reactions.

In some doped perovskite structures, it is known that diffusion of oxygen happens rather frequently. At the same time, we may expect motion of metal atoms via a process exchanging metallic atoms. The process would be enhanced, if oxygen vacancies are created in the perovskite host crystal. This scenario might support the above model of self-regenerating perovskite catalysts. For realization of high efficiency in the three-way catalytic functions with

\footnotetext{
*E-mail address: hkizaki@aquarius.mp.es.osaka-u.ac.jp
} 
no deterioration, however, we should consider another model of the self-regenerating catalyst. The new model should explain some mysteries known experimentally in the perovskite catalyst. The self-regeneration can happen, only when the host perovskite lattice structure maintains its essential structure. We need to know why the redox reaction keeps its cycle in a redox environment changing in a frequency of about a few hertz $(1 \sim 4 \mathrm{~Hz})$. Thus we need to understand motion of precious metal atoms in a rather hard oxide. Once melting happens in some process of catalytic reaction, we again cannot escape from agglomeration of metallic atoms leading to deterioration. If these problems are solved, we can hope to design much efficient catalysts from the knowledge of nano-scale structure of solid catalysts.

To answer these questions, we propose a new model of the self-regenerating perovskite catalyst based on the spinodal nano-decomposition (SND). ${ }^{12-16)}$ In several covalent and ionic materials, it is known that growth of nano-scale structures happens in a thermal non-equilibrium synthesis process of the materials due to SND. One of the most remarkable example is SND appearing in diluted magnetic semiconductors. The mechanism of SND was first clarified theoretically. ${ }^{12)}$ Later, many experimental evidences supporting appearance of SND were found. ${ }^{17,18)}$ Actually, enhancement of the blocking temperature appears in the super-paramagnetic substance, in which ferromagnetic hysteretic behavior in magnetization process happens. Novel functional device structures and new functional materials are proposed based on SND. ${ }^{19,20)}$ In the perovskite catalysts, due to difference in the solubility of metallic atoms in the host matrix, there can happen SND. In $\mathrm{LaFeO}_{3}$, for example, solubility of precious metal is low and it should be different for $\mathrm{Pd}, \mathrm{Rh}$, and Pt. Once the phenomenon occurs, the above puzzling problems are naturally solved as shown in this paper.

Our purpose of the present study is thus summarized in the following steps. At first, to support occurrence of SND, we will give the first-principles simulation using the Korringa-KohnRostoker method with the coherent-potential approximation (KKR-CPA). ${ }^{21)}$ The numerical data supports SND at a reasonable chemical composition and at a plausible temperature. We will thus proceed to consider a physical picture concluded by SND, explaining the SND model in detail. Phenomenological solutions for the problems in perovskite catalysts are given. Functionality of the nano-scale catalyst is a key point, which gives a natural explanation of the self-regenerating catalysts. Several candidate materials for the new three-way catalysts are proposed based on the materials design using SND.

We consider $\mathrm{La}\left(\mathrm{Fe}_{1-x} \mathrm{Pd}_{x}\right) \mathrm{O}_{3}$ as the typical perovskite catalyst. To explore material dependence, $\mathrm{La}\left(\mathrm{Fe}_{1-x} \mathrm{Rh}_{x}\right) \mathrm{O}_{3}$ is also examined. In these examples, the host material is $\mathrm{LaFeO}_{3}$. The position of doped precious metal is known to be at the B site of the perovskite structure. ${ }^{2,7,24)}$

In covalent ionic crystals, the spinodal decomposition or the phase separation often happens depending on the composition and the temperature. For $\mathrm{La}\left(\mathrm{Fe}_{1-x} \mathrm{Pd}_{x}\right) \mathrm{O}_{3}$, depending 
on synthesis processes, the sample can microscopically decompose into $\mathrm{LaFeO}_{3}$ and $\mathrm{LaPdO}_{3}$. When SND happens, spatial inhomogeneity in distribution of Pd atoms occurs but the crystal structure itself is maintained. X-ray diffraction will see average crystallinity only, if the size of each nano-scale structure is less than $1 \mathrm{~nm}$. To characterize the distribution and to discriminate SND from the phase separation, we are required to utilize electron energy loss spectroscopy (EELS) or energy dispersive X-ray spectroscopy (EDXS). ${ }^{17,18)}$ To show a support for this message, we have performed estimation of the mixing energy of $\mathrm{LaFeO}_{3}$ and $\mathrm{LaPdO}_{3}$, or that of $\mathrm{LaPdO}_{3}$ and $\mathrm{LaRhO}_{3}$. The mixing energy $\Delta E$ is given for $\mathrm{La}\left(\mathrm{Fe}_{1-x} \mathrm{Pd}_{x}\right) \mathrm{O}_{3}$ as $\Delta E(x)=E\left(\mathrm{La}\left(\mathrm{Fe}_{1-x} \mathrm{Pd}_{x}\right) \mathrm{O}_{3}\right)-\left\{(1-x) E\left(\mathrm{LaFeO}_{3}\right)+x E\left(\mathrm{LaPdO}_{3}\right)\right\}$, where $E(\mathrm{M})$ is the total energy of a material $\mathrm{M}$. If $\Delta E$ is positive, the system has a tendency toward spinodal decomposition (or phase separation), while negative $\Delta E$ suggests that the system favors a homogeneous mixing. The actual simulation was done utilizing the KKR-CPA program package MACHIKANEYAMA2002 developed by H. Akai. ${ }^{22)}$

In Fig. 1 (a) and (b), we show the results for $\mathrm{La}\left(\mathrm{Fe}_{1-x} \mathrm{Pd}_{x}\right) \mathrm{O}_{3}$ and $\mathrm{La}\left(\mathrm{Fe}_{1-x} \mathrm{Rh}_{x}\right) \mathrm{O}_{3}$, respectively. The obtained density of states for a $5 \% \mathrm{Pd}$-doped $\mathrm{LaFeO}_{3}$ system is shown in Fig. 1 (c). At zero temperature, both of the results indicate tendency to the spinodal decomposition. As shown in the figures, the mixing energies show strong concavity as a function of $\mathrm{Pd}$ or $\mathrm{Rh}$ impurity concentration. To show the temperature dependence, we introduce the mixing entropy to have a free energy as $F=\Delta E(x)-T S$ with $S=-k_{B}[x \log x+(1-x) \log (1-x)]$. In this estimation, some temperature effects including the volume expansion and lattice deformation, kinetics of atoms leading to atomic diffusion, defect formation, and crack creation, are not considered. However, the present test should be the first prior test to judge whether mixing happens in equilibrium or not. The results shown in Fig. 1 (a) and (b) indicate clearly the phase separation $\left(\partial^{2} F / \partial x^{2}>0\right)$ or spinodal decomposition $\left(\partial^{2} F / \partial x^{2}<0\right)$ at a finite temperature plausible for the ordinal synthesis process. The free energy curves suggest that, only above a rather high temperature $(T>3000 \mathrm{~K}$ for $\mathrm{Pd}(x=10 \%)$ and $T>2000 \mathrm{~K}$ for $\mathrm{Rh}$ $(x=10 \%))$, mixing may happen.

If the synthesis is done in non-equilibrium condition, $\mathrm{Pd}$ and $\mathrm{Rh}$ could be dispersed in the host matrix. However, our simulation strongly suggests that the solubility of $\mathrm{Pd}$ and $\mathrm{Rh}$ in $\mathrm{LaFeO}_{3}$ is small and SND should occur in the concave region of $F\left(\partial^{2} F / \partial x^{2}<0\right)$, when calcination is performed. Depending on the heat condition and the calcination time, the size of segregated precious metal oxide grain due to SND might be controlled.

Our simulation results show that the tendency towards the spinodal decomposition is less for the Rh-compound than the $\mathrm{Pd}$-compound. Actually, the value of $\Delta E(x)$ is about $15 \%$ smaller for the Rh-compound than that of the Pd-compound. Larger mixing energy for $\mathrm{La}\left(\mathrm{Fe}_{1-x} \mathrm{Pd}_{x}\right) \mathrm{O}_{3}$ indicates that spinodal decomposition should be more serious for this material than for $\mathrm{La}\left(\mathrm{Fe}_{1-x} \mathrm{Rh}_{x}\right) \mathrm{O}_{3}$. Indeed, we can clearly see in Fig. 1 (a) and (b) at $\mathrm{T}=1000 \mathrm{~K}$ 
that $\partial^{2} F / \partial x^{2}<0$ for $\mathrm{Pd}(x=5 \%)$ increasing SND, however, $\partial^{2} F / \partial x^{2}>0$ for $\mathrm{Rh}(x=$ $5 \%$ ) indicating no spinodal decomposition. This result is consistent with the experimental observations. The system in $\mathrm{La}\left(\mathrm{Fe}_{1-x} \mathrm{Rh}_{x}\right) \mathrm{O}_{3}$ favors a homogeneous mixing, in which $\mathrm{Rh}$ atoms tends to disperse in $\mathrm{LaFeO}_{3}$ matrix and therefore less effective for the catalytic reactions than $\mathrm{La}\left(\mathrm{Fe}_{1-x} \mathrm{Pd}_{x}\right) \mathrm{O}_{3}$, as known in the experiment. ${ }^{7)}$

We now discuss relevance of SND for the realization of the regenerating catalytic function, which makes us possible to design new three-way catalysts. Before explaining the SND model, let us consider what happens if the catalysts are made from uniformly dispersed precious metals in the synthesis process and if SND does not occur. Then diffusion of the precious metal atoms from the bulk to the reacting surfaces are required for efficient redox reactions. Then, segregation of precious metal atoms is expected. Nano-particles found in XAFS experiments have been thought to be created by this process in the uniformly distributed model. Once the formation of precious metal particles at the reaction surface occurs, leaving defective B-sites in the host, agglomeration of the metal particles and destabilization of the host matrix are inevitable, which leads to some difficulty in understanding the self-regeneration.

When SND happens in a synthesis process, nano-catalysts are created in the host matrix. (Fig. 2) In the redox reaction, only the nano-catalyst particles react with oxygen atoms going out and into the nano-catalysts, keeping the host matrix stable. The reaction is enhanced due to high concentration of precious (or relevant non-precious) metal atoms at the reacting surfaces. In the SND model, thus we can naturally explain stability of the catalysts found in the experiment. ${ }^{23)}$

The SND model has a big advantage to understand the fast catalytic activity. ${ }^{24-26)}$ Since the reactive nano-catalyst particles are formed at the reaction surfaces, we can expect very fast start-up for the catalytic activity. If the host matrix is enough stable, we can hope high endurance against the heat, since the reacting nano-catalysts keep their size in nano-scale. In addition, the nano-scale catalyst particles have quantum effects enhancing the catalytic activity. Catalytic reactions would be affected by discretization of electronic energy levels. In nano-catalysts embedded in a host matrix, there would be appearance of different valence states in a reasonable energy range around the Fermi level, which would be controlled by conditions of the gas phase.

Following the SND model, we can readily design many functional nano-catalysts utilized in a wide area of application for the perovskite catalysts. ${ }^{27-29)}$ An important selection rule is given by solubility of relevant metal atoms in a host matrix. We should select relevant $3 d$ transition elements for the reduction or the abandonment of the precious metal. Important candidates should be $\mathrm{Mn}, \mathrm{Fe}, \mathrm{Co}, \mathrm{Ni}$, and $\mathrm{Cu}$. Consideirng the solubility, possible materials are, $\mathrm{Ca}\left(\mathrm{Ti}_{1-x} \mathrm{M}_{x}\right) \mathrm{O}_{3}, \operatorname{Sr}\left(\mathrm{Ti}_{1-x} \mathrm{M}_{x}\right) \mathrm{O}_{3}, \mathrm{Ba}\left(\mathrm{Ti}_{1-x} \mathrm{M}_{x}\right) \mathrm{O}_{3}, \mathrm{Ca}\left(\mathrm{Zr}_{1-x} \mathrm{M}_{x}\right) \mathrm{O}_{3}, \operatorname{Sr}\left(\mathrm{Zr}_{1-x} \mathrm{M}_{x}\right) \mathrm{O}_{3}$, and $\mathrm{Ba}\left(\mathrm{Zr}_{1-x} \mathrm{M}_{x}\right) \mathrm{O}_{3}$ with $\mathrm{M}=\mathrm{Mn}, \mathrm{Fe}, \mathrm{Co}, \mathrm{Ni}, \mathrm{Cu}$, and $\mathrm{Zn}$. If we inversely consider functions 
of elements, the next structure might be another solution: $\mathrm{La}\left(\mathrm{Fe}_{1-x} \mathrm{M}_{x}\right) \mathrm{O}_{3}$ with $\mathrm{M}=\mathrm{Ti}$ and V.

\section{Acknowledgement}

This work was partially supported by the Elements Science and Technology Project, Grant-in-Aid for Scientific Research from the Japan Society for the Promotion of Science and the Ministry of Education, Culture, Sports, Science and Technology (MEXT), Global Center of Excellence Program by MEXT, New Energy and Industrial Technology Development Organization Program, and Japan Science and Technology Agency Program. 

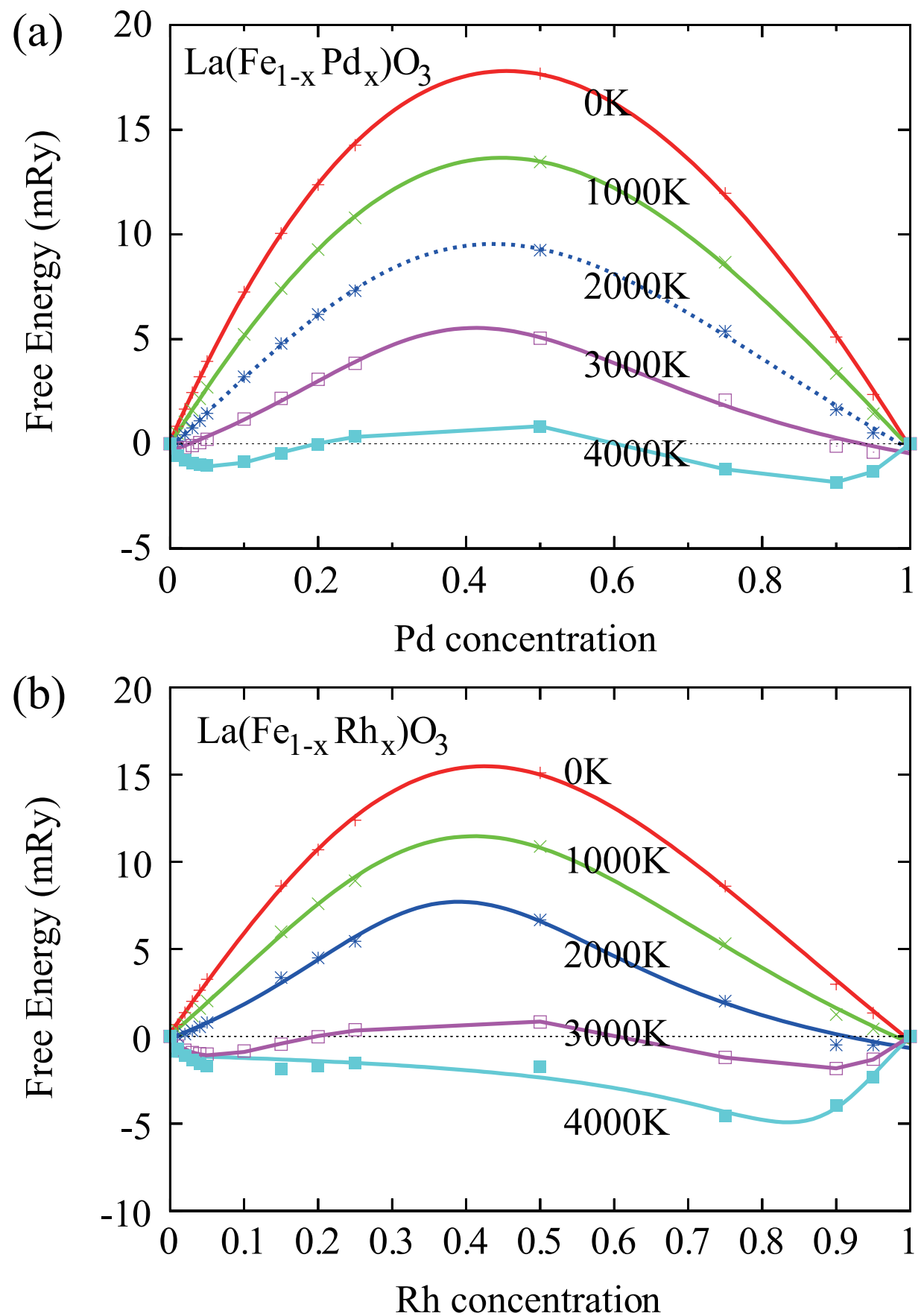


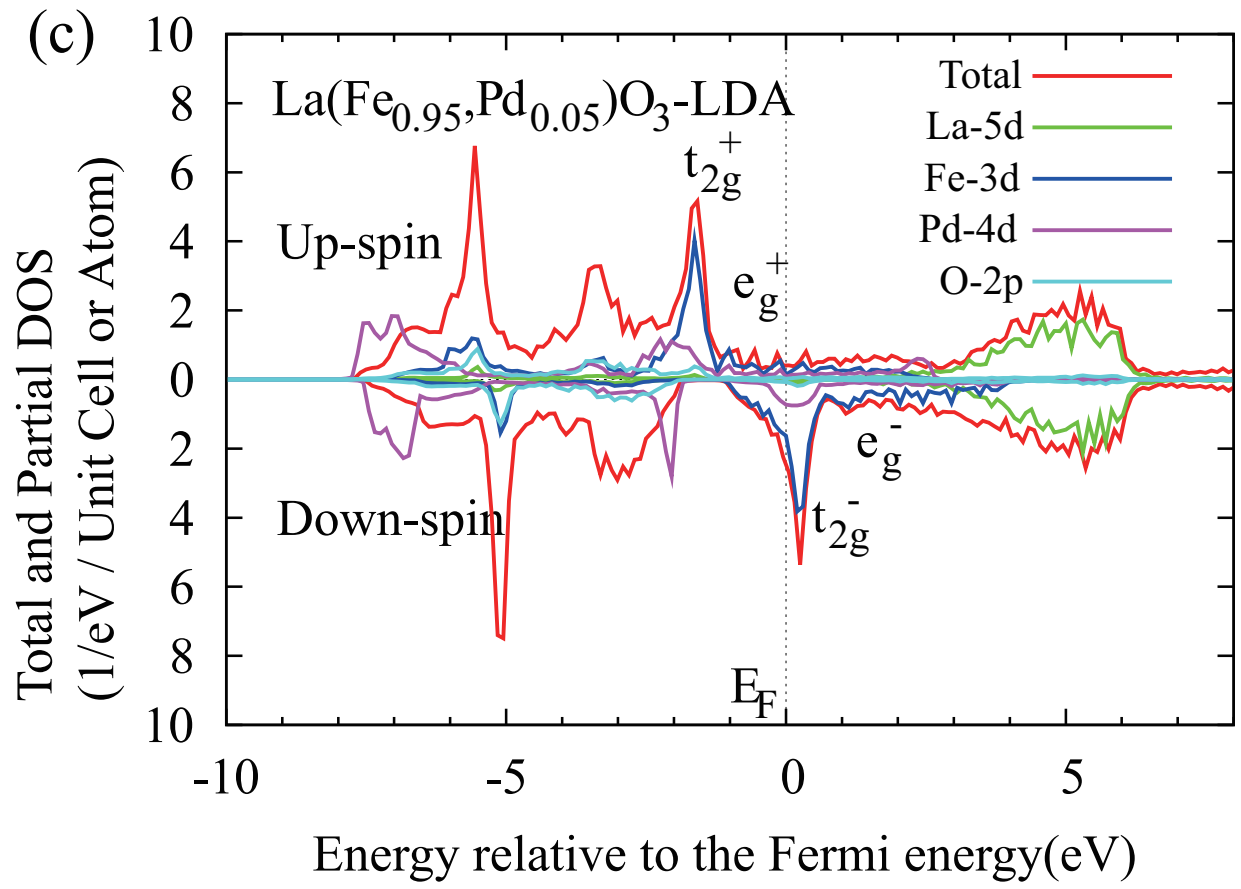

Fig. 1. Calculated free energies of (a) $\mathrm{La}\left(\mathrm{Fe}_{1-x} \mathrm{Pd}_{x}\right) \mathrm{O}_{3}$ and (b) $\mathrm{La}\left(\mathrm{Fe}_{1-x} \mathrm{Rh}_{x}\right) \mathrm{O}_{3}$. The free energy is calculated as a function of concentration of the precious metal. The temperature dependence is obtained by introducing the mixing entropy. In the panel (c), calculated total and partial density of states for $\mathrm{La}\left(\mathrm{Fe}_{0.95} \mathrm{Pd}_{0.05}\right) \mathrm{O}_{3}$ are shown. 

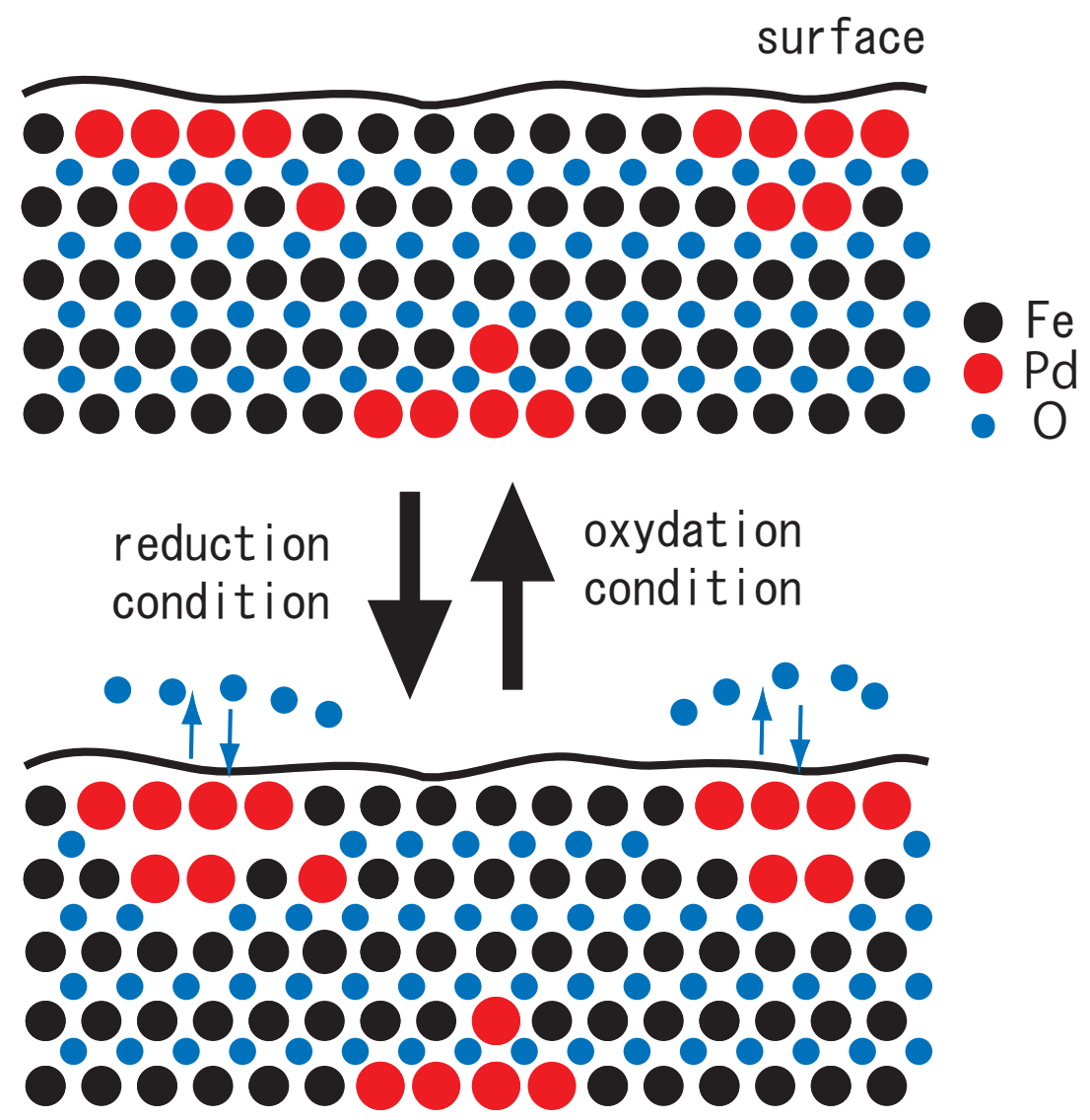

Fig. 2. Schematic pictures of the spinodal nano-decomposition model of the self-regenerating perovskite catalysts. Black, red, and blue atoms are iron, palladium, and oxygen atoms, respectively. In the redox reaction, motion of atoms shown in each figure should happen. La-O bond is strong and stable in the reduction condition. Therefore, we do not show La-O in this schematic picture. 


\section{References}

1) H. Tanaka, I. Tan, M. Uenishi, M. Kimura, K. Dohmae: Topics in Catalysis, 16/17, 63 (2001).

2) Y. Nishihata, J. Mizuki, T. Akao, H. Tanaka, M. Uenishi, M. Kimura, T. Okamoto, N. Hamada: Nature, 418, 164 (2002).

3) H. Tanaka, M. Taniguchi, N. Kajita, M. Uenishi, I. Tan, N. Sato, K. Narita, M. Kimura: Topics in Catalysis, 30/31, 389 (2004).

4) Y. Nishihata, J. Mizuki, H. Tanaka, M. Uenishi, M. Kimura: J. Phys. Chem. of Solid, 66, $274(2005)$.

5) M. Uenishi, H. Tanaka, M. Taniguchi, I. Tan, Y. Sakamoto, S. Matsunaga, K. Yokota, T. Kobayashi: Applied Catalysis A: General, 296, 114 (2005).

6) H. Tanaka, I. Tan, M. Uenishi, M. Taniguchi, M. Kimura, Y. Nishihata, J. Mizuki: J. Alloys Compd., 408-412, 1071 (2006).

7) H. Tanaka, M. Taniguchi, M. Uenishi, et al.: Ang. Chem. Int. Ed. 45, 5998 (2006).

8) H. Tanaka, M. Uenishi, M. Taniguchi, et al.: Catalysis Today 117, 321 (2006).

9) H. Tanaka, I. Tan, M. Uenishi, M. Taniguchi, Y. Nishihata, J. Mizuki: Key Engineering Materials, 317-318, 827 (2006).

10) I. Tan, M. Taniguchi, H. Tanaka, M. Uenishi, N. Kajita, Y. Nishihata, J. Mizuki, K. Niihara: Key Engineering Materials, 317-318, 833 (2006).

11) M. Taniguchi, H. Tanaka, M. Uenishi, et al.: Topics in Catalysis, 42-43, 367 (2007).

12) K. Sato, H. Katayama-Yoshida, and P.H. Dederichs: Jpn. J. Appl. Phys. 44 (2005) L948.

13) T. Fukushima, K. Sato, H. Katayama-Yoshida, and P.H. Dederichs: Jpn. J. Appl. Phys. 45 (2006) L416.

14) K. Sato, T. Fukushima, H. Katayama-Yoshida: Jpn. J. Appl. Phys. 46 (2007) L682.

15) K. Sato, T. Fukushima, H. Katayama-Yoshida: J. Phys. Condens. Matter, 19 (2007) 365212 .

16) K. Sato, T. Fukushima, H. Katayama-Yoshida: Jpn. J. Appl. Phys. 46 (2007) L1120.

17) L. Gu, S.Y. Wu, H.X. Liu, R.K. Singh, N. Newman, and D.J. Smith: J. Magn. Magn. Mater. 290-291 (2005) 1395.

18) M. Jamet, A. Barski, T. Devillers, V. Poydenot, R. Dujardin, P. Bayle-Guillemaud, J. Rothman, E. Bellet-Amalric, A. Marty, J. Cibert, R. Mattana, and S. Tatarenko: Nat. Mater. 5 (2006) 653.

19) H. Katayama-Yoshida, K. Sato, T. Fukushima, M. Toyoda, H. Kizaki, V.A. Dinh, and P.H. Dederichs: J. Magn. Magn. Mater. 310 (2007) 2070. 
20) H. Katayama-Yoshida, K. Sato, T. Fukushima, M. Toyoda, H. Kizaki, V.A. Dinh, and P.H. Dederichs: Phys. Status Solidi A 204 (2007) 15.

21) H. Akai and P.H. Dederichs: Phys. Rev. B 47 (1993) 8739.

22) H. Akai: http://sham.phys.sci.osaka-u.ac.jp/kkr/

23) I. Tan, H. Tanaka, M. Uenishi, et al.: J. Ceramic Soc. Jpn. 113, 71 (2005).

24) Y. Nishihata, J. Mizuki, H. Tanaka, M. Uenishi, M. Taniguchi, K. Kato, T. Uruga: The ECS Proceedings Volume from the Symposium on High Temperature Corrosion and Materials Chemistry V, 182 (2004).

25) M. Uenishi, M. Taniguchi, H. Tanaka, M. Kimura, Y. Nishihata, J. Mizuki, T. Kobayashi: Applied Catalysis B: Environmental, 57, 267 (2005).

26) M. Uenishi, H. Tanaka, M. Taniguchi, et al.: Catalysis Commun. 9, 311 (2008).

27) H. Tanaka, N. Mizuno, M. Misono: Applied Catalysis A: General, 244, 371 (2003).

28) S. Lohmann, S. P. Andrews, B. J. Burke, M. D. Smith, J. P. Attfield, H. Tanaka, K. Kaneko, S. V. Ley: Synlett, 8, 1291 (2005).

29) S. P. Andrews, A. F. Stepan, H. Tanaka, S. V. Ley, M. D. Smith: Adv. Synth. Catal., 347, 647 (2005). 